\title{
INDICADORES TURÍSTICOS: OFERTA Y DEMANDA DE LA CIUDAD PATRIMONIAL DE CUENCA - ECUADOR
}

\author{
Ana Lucia Serrano* \\ Elena Villafuerte Pucha**
}

\begin{abstract}
Resumen: El objetivo de este estudio fue identificar los IODT del cantón Cuenca a través de la recopilación de información primaria y secundaria para su posterior implementación y manejo a fin de caracterizar el mercado del turismo local y determinar la relación con el macro entorno turístico. Metodológicamente se utilizó un enfoque cuantitativo con alcance descriptivo mediante encuestas cerradas a través de un muestreo no probabilístico por conveniencia, 119 establecimientos de alojamiento de un total de 223, y 2.000 turistas entre nacionales y extranjeros. En relación al alojamiento se obtuvo: los visitantes pernoctan un promedio de dos noches; hay segmentos de turistas con motivación de ocio y corporativos siendo estos clientes habituales que visitan Cuenca por trabajo; las necesidades más apremiantes en orden de importancia son: capacitación en servicio al cliente, manejo de medios digitales, los cargos ejecutivos necesitan instrucción superior. En relación a visitantes se obtuvo: género masculino (55.26\%) y femenino (44,24\%); edad entre 26 - 36 años; se autodefinen en su mayoría como turistas culturales y de experiencias; el tiempo de pernoctación es de 2 o 3 noches; la procedencia principal de los turistas es en orden de mayor cantidad: Estados Unidos, Colombia, Argentina y Alemania. Este análisis sugiere orden en la categorización y clasificación de alojamiento, sin embargo, es un enclave de oportunidades para las alianzas que se pueden generar entre los actores del sector turístico.
\end{abstract}

Palabras clave: Indicadores turísticos; Ocupación hotelera; Visitantes; Oferta y demanda turística; Cuenca (Ecuador).

\section{INDICADORES TURÍSTICOS: OFERTAE DEMANDA DACIDADE PATRIMONIAL DE CUENCA-ECUADOR}

Resumo: O objetivo deste estudo foi identificar o IODT da província de Cuenca, através da coleta de informações primárias e secundárias para sua posterior implementação e gerenciamento, a fim de caracterizar o mercado turístico local e determinar a relação com o macro ambiente do turismo. Metodologicamente, utilizou-se a abordagem quantitativa com escopo descritivo por meio da aplicação de questionários junto a uma amostra não probabilística e por conveniência com 119 estabelecimentos de alojamento (num total de 223) e junto a 2.000 turistas, entre nacionais e estrangeiros. Em relação à acomodação foi verificou-se que: os visitantes pernoitam em média 2 noites; há segmentos turísticos com motivação de lazer e corporativo, sendo esses clientes regulares, e que visitam Cuenca a trabalho; as necessidades mais urgentes em ordem de importância são: treinamento em atendimento ao cliente, gerenciamento de mídia digital, e ensino superior para cargos executivos. Em relação aos visitantes, verificou-se que: 55,26\% são do sexo masculino e 44,24\% feminino, com idade entre 26 a 36 anos; auto definindo-se como turistas culturais e de experiências; com tempo de estadia de 2 a 3 noites; e a principal fonte de turistas (em ordem decrescente): Estados Unidos, Colômbia, Argentina eAlemanha. Esta análise sugere ordem na categorização e classificação de acomodação, no entanto, é um enclave de oportunidades para as alianças que podem ser geradas entre os atores do setor de turismo.

Palavras-chave: Indicadores turísticos; Ocupación hotelera; Visitantes; Oferta y demanda turística; Cuenca (Ecuador).

\section{TOURISM INDICATORS: SUPPLY AND DEMAND FOR THE PATRIMONIALCITY OFCUENCA-ECUADOR}

\begin{abstract}
The objective of this study was to identify the IODT of the canton of Cuenca through the collection of primary and secondary information for its subsequent implementation and management in order to characterize the local tourism market and determine the relationship with the tourism macro-environment. Methodologically, a quantitative approach with descriptive scope was used through closed surveys through a non-probabilistic convenience sampling, 119 accommodation establishments out of a total of 223, and 2,000 tourists between nationals and foreigners. In relation to accommodation was obtained: visitors spend an average of two nights; there are tourist segments with leisure and corporate motivation being these regular customers who visit Cuenca for work; The most pressing needs in order of importance are: training in customer service, management of digital media, executive positions need higher education. In relation to visitors, we obtained: male (55.26\%) and female (44.24\%); Age between 26 - 36 years; they define themselves mostly as cultural tourists and as experiences; Overnight time is 2 or 3 nights; The main source of tourists is in order of more: United States, Colombia, Argentina and Germany. This analysis suggests order in the categorization and classification of accommodation, however, it is an enclave of opportunities for the alliances that can be generated among the actors of the tourism sector.
\end{abstract}

Keywords: Tourism indicators, hotel occupancy, visitors, supply and demand, Cuenca (Ecuador).

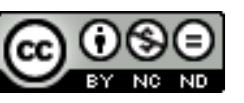

Licenciada por Creative Commons Atribuição Não Comercial / Sem Derivações/4.0/Internacional
* Doctorado, en curso, en Satisfacción Laboral con énfasis en el capital humano, Universidad de Córdoba/España. Docente y Directora del Centro de Investigación de la Facultad de Hospitalidad de la Universidad de Cuenca/Ecuador. Leva 11 años como docente titular y en 2015 crea el primer grupo de investigación de la Facultad de Ciencias de la Hospitalidad, ha participado en varios proyectos de investigación, autora y coautora de artículos cientíicos. [ana.serrano@ucuenca.edu.ec]

**Egresada de la Escuela de Hotelería de la Facultad de Ciencias de la Hospitalidad de la Universidad de Cuenca, se ha desempeñado como ayudante de investigación en la Facultad de Ciencias de la Hospitalidad desde el año 2015 hasta el 2017. [elena.villafuerte@ucuenca.ec] 


\section{INTRODUCCIÓN}

La oferta turística tal como el alojamiento pertenece a la planta turística de un destino y aporta significativamente a la competitividad y desarrollo turístico, no obstante, a nivel mundial ha experimentado avances en su organización interna y externa, por consiguiente, afrontan nuevos retos para su gestión (Boullón, 2006); además, en torno a la planta turística están los atractivos turísticos y entre estos aspectos a más de otros interactúa el turista.

En la actualidad el turismo es un fenómeno que tiene distintas consecuencias económicas relacionadas con el desarrollo, siendo dependiente de factores: sociales, políticos, geográficos, económicos, culturales, la globalización, entre otros, y, a diferencia de la industria basa su accionar en gran parte respecto a los servicios por lo que puede ser considerado como un producto de exportación reduciendo de cierta manera la vulnerabilidad países en vías de desarrollo a la explotación de recursos, en algunos de estos países existe un incremento de turistas en los últimos años, lo cual ha hecho que los gobiernos consideren esta actividad dentro de sus políticas como estrategia de desarrollo (Zhao \& Li, 2006).

En Costa Rica, Colombia y Perú se evidencia incremento de turistas en los dos últimos años (2015, 2016) (Instituto Costarricense de Turismo, 2017; DANE, 2017; Mincetur 2017), sin embargo, en Ecuador el comportamiento ha sido distinto en 2015 aproximadamente 1,600.000, en 2016 aproximadamente 1,400.00, (Ministerio de Turismo, 2017), por lo que se sugiere analizar la dinámica entre oferta y demanda y de aquello no está exenta la ciudad de Cuenca puesto que en 2009 ingresaron 240.000 turistas, (Fundación Municipal Turismo para Cuenca \& Ministerio de Turismo, 2011), la cual posee distintas distinciones como por ejemplo Patrimonio Cultural de la Humanidad (1999) entre otras (Fundación Municipal Turismo para Cuenca, 2017); además International Living calificó a Cuenca como el "Paraíso para jubilados retirados" (Ministerio de Turismo, 2013).

Entonces es primordial establecer indicadores de oferta y demanda aplicado a la realidad del sitio en virtud del comportamiento de las empresas de alojamiento y el movimiento turístico en la ciudad con la intención de generar herramientas para un desarrollo armónico basado en la investigación.

Tomando en cuenta este contexto, el presente artículo, que es derivado del proyecto de investigación "Creación, implementación y manejo de indicadores turísticos para el cantón Cuenca", de la Facultad de Ciencias de la Hospitalidad de la Universidad de Cuenca, tiene como objetivo general identificar indicadores de oferta y demanda turística del cantón Cuenca a través de la recopilación de información primaria y secundaria para su posterior implementación y manejo a fin de caracterizar el mercado del turismo local y determinar la relación con el macro entorno turístico.

En cuanto a los objetivos específicos se plantea: 1) Exponer la dinámica del mercado turístico local, las preferencias de la demanda turística con particular énfasis en el consumo de alimentos y la oferta turística global, 2) Analizar comparativamente el destino Cuenca con el macro entorno turístico nacional e internacional, y, 3) Plantear la vinculación de la academia en la parte investigativa, además se buscó la alianza estratégica con el sector público y privado.

\section{MARCO TEÓRICO}

El turismo, en su definición y práctica, está íntimamente relacionado con el movimiento de las personas a lugares distintos a su residencia habitual, quienes, de acuerdo al tiempo de estancia pueden ser visitantes excursionistas, residentes o no residentes (Organización Mundial del Turismo, 2013). Dicha movilidad responde a la necesidad humana de desplazamiento en búsqueda de libertad para conocer el mundo e interactuar cara a cara con otras culturas (Dachary, 2015; Campodónico, 2016) que es satisfecha a través de la actividad turística, con la cual los individuos gozan de una amplia oferta para descubrir tantos lugares que diversifican las experiencias de consumo (Dachary, 2015) y que marcan pautas en la demanda.

El turismo en la última década, a pesar de las crisis económicas que han vivido muchos países, ha mantenido un desarrollo creciente que lo ubica como uno de los sectores más importantes y sólidos de la economía mundial, incluso superior a las exportaciones de petróleo. Tal es su desarrollo que, según la Organización Mundial del Turismo (2017), aporta el 10\% del Producto Interno Bruto (PIB) mundial, genera exportaciones por más de 1.5 billones de dólares norteamericanos y constituye el 7\% del comercio internacional (Santiago, Romero, \& Álvarez, 2017).

En el caso de Ecuador, los resultados de la investigación realizada por Ayaviri, Quispe y Sánchez 
(2017) para la estimación de la demanda en los países de la Comunidad Andina de Naciones, reflejan que Ecuador es el país con mayor flujo turístico receptivo y estimación de la demanda internacional. Ecuador ha logrado un crecimiento constante en el volumen de arribos internacionales, superando el millón y medio de visitantes, con una tasa de ocupación promedio superior al $50 \%$ y con ingresos que ascienden a 1075.5 millones de USD al año 2016 (Santiago, Romero, \& Álvarez, 2017). Lo que, en consecuencia, favorece al turismo de la ciudad de Cuenca, si consideramos que es la tercera ciudad más importante del Ecuador, la que además ha visto incrementados sus ingresos por concepto de turismo a partir de su declaratoria como Patrimonio Cultural de la Humanidad por la UNESCO en el año 1999.

En este contexto, ciertamente el turismo es un fenómeno social y cultural que tiene efectos en la economía de los lugares visitados (Organización Mundial del Turismo, 2013), su impacto se mide a través de indicadores turísticos y datos estadísticos, como el conteo de unidades de hospedaje (Campodónico, 2016). La información estadística sobre el turismo es importante para la gestión sostenible del destino y es una sólida herramienta para el análisis de las tendencias actuales y futuras del turismo además fundamental para la toma de decisiones por parte de gobiernos locales (Saeteros, Da Silva, \& Calles, 2017).

Los indicadores turísticos dan cuenta de la planificación turística, que es la organización del destino turístico para el aprovechamiento de sus fortalezas, que es un requerimiento fundamental para alcanzar el éxito en el desarrollo, gestión y manejo de la actividad a escala internacional, nacional y/o local porque garantiza la correcta integración del turismo en la economía, la sociedad, la cultura y el medio ambiente; así como la adecuada administración de la oferta y la satisfacción de la demanda (Organización Mundial del Turismo, 2004).

Desde el punto de vista conceptual, la oferta turística son los bienes y sus satisfactores que se encuentran en el lugar a donde los consumidores se desplazan, donde se desarrolla la actividad turística, y que influye directamente en sus preferencias y selección del destino. (Ivanov \& Webster, 2007; Ayaviri, Quispe, \& Sánchez, 2017). Son los bienes y servicios disponibles, capacidad técnica para satisfacer los deseos de los visitantes, precios de mercado e ingresos (Ayaviri, Quispe, \& Sánchez, 2017). Por ello, el estudio de la oferta es imprescindible, sobre todo si se tiene en cuenta que actualmente la oferta turística crece a ritmos más elevados que la demanda (Flores \& Barroso, 2012).

Entre los elementos que conforman la oferta turística está la hotelería, uno de los servicios turísticos básicos que hace posible la actividad turísticas, y que, hoy por hoy está caracterizada por las grandes cadenas hoteleras y hoteles de lujo (Jímenez, 2008), que responden a las necesidades actuales de turistas más experimentados y "globalizados" que son más exigentes y selectivos a la hora de planificar sus vacaciones (Wallingre, 2005).Así, la ocupación hotelera en Ecuador, según la encuesta coyuntural de alojamiento aplicada por la Coordinación General de Estadística del Ministerio de turismo en Ecuador, en el año 2016 indica que la tasa de ocupación promedio para instalaciones hoteleras de primera categoría fue de $43 \%$, mientras para las instalaciones hoteleras de lujo ascendió al 65\% (Santiago, Romero, \& Álvarez, 2017).

La adaptación a las necesidades actuales de los turistas constituye también un elemento básico en la planificación de la oferta, en ella, tiene un papel fundamental el profesionalismo del personal hotelero, puesto que, las mejoras en las tecnologías de la información y la comunicación y su aplicación al turismo han provocado un cambio en las funciones tradicionales de producción, comercialización y distribución del sector, aumentando el poder de la demanda en el mercado turístico (Flores \& Barroso, 2012). De ahí la necesidad de ofertar servicios que sean operados por personal capacitado para atender a una demanda que, debido a los cambios demográficos, al aumento de la esperanza de vida y a la disminución de la tasa de natalidad, incrementa en edad y provoca que esta sea más experimentada y exigente (Flores \& Barroso, 2012).

La demanda turística comprende el movimiento de individuos hacia los diversos destinos y su estancia en los mismos (características de alojamiento y actividades realizadas en el destino); así como, todos los bienes y servicios requeridos por el visitante durante el desplazamiento de su residencia hacia el destino, y su estadía (Flores \& Barroso, 2012). La demanda turística es la cantidad de producto turístico que los potenciales consumidores están dispuestos a adquirir a un precio en un momento determinado (Ayaviri, Quispe, \& Sánchez, 2017). 
Entre las características de la demanda turística mundial, tanto las tasas de crecimiento de las llegadas de turistas internacionales como las de los ingresos toman valores positivos desde principios de la década de los sesenta, ya que es a partir de este período cuando comienza a desarrollarse el denominado "turismo de masas" (Flores \& Barroso, 2012) como consecuencia, entre otros, de los siguientes factores:

- El Pacto Internacional de los Derechos Sociales, Económicos y Culturales de principios de los años setenta, donde se consagra el derecho al turismo como un hecho social, y el derecho al descanso como una necesidad humana (Dachary, 2015).

- La política gubernamental que reconoce el derecho de los trabajadores a vacaciones remuneradas (Flores \& Barroso, 2012).

- Los avances en el desarrollo y bienestar del ser humano después de la II Guerra Mundial, tales como: el aumento de la esperanza de vida de la población, el adelanto en la edad de jubilación y la progresiva reducción de la jornada laboral, lo cual aumenta el tiempo que tienen las personas para viajar (Flores \& Barroso, 2012).

- El movimiento de millones de personas en cortos períodos de tiempo que ha disminuido la estancia del turista en el destino, aumentando su número de salidas (Flores \& Barroso, 2012).

- Fragmentación de las vacaciones que demandan viajes especializados y temáticos por nichos de mercado más amplios que viajan a más lugares y buscan más experiencias (Flores \& Barroso, 2012).

- El acceso a tanta oferta y libertad para elegir que le permite al consumidor viajar y descubrir sitios culturales y degustar platos exóticos con distancias y espacio que se han reducido gracias al avance en transportación (Dachary, 2015).

Así, la planificación turística incorpora la complementariedad y la interacción de la oferta y la demanda para tomar decisiones gerenciales oportunas y competitivas en la región para la gestión del destino (Ayaviri, Quispe, \& Sánchez, 2017).

\section{METODOLOGÍA}

La metodología utilizada en este estudio tiene enfoque cuantitativo de alcance descriptivo para lo cual se ha llevado un proceso de capacitación para la toma correcta de datos y tabulación.

Los establecimientos de hospedaje se clasificaron en: hoteles, hoteles boutique, hostales, hosterías, casa de huéspedes, pensiones, apartamentos turísticos y refugios. Respecto a los establecimientos de hospedaje la toma de información fue con un muestreo al azar del inventario establecido por el por el Ministerio de Turismo, presentando un error del $2 \%$.

En el caso de los turistas se estableció 12 conglomerados y toma de muestra al azar por lo cual los datos levantados fueron a partir de un muestreo aleatorio no probabilístico por conveniencia, con un $5,5 \%$ de error y 95\% nivel confianza. También se realizó una selección de diversos atractivos y lugares de concentración de turistas, nacionales y extranjeros, que visitan la ciudad de Cuenca, mediante este proceso se estableció los puntos de levantamientos.

Para establecer el formulario de encuesta se realizó una revisión bibliográfica tal como: legislación, políticas, indicadores turísticos planteados por la OMT, catastro turístico ecuatoriano, entre otros. La plantilla de encuesta fue elaborada, socializada y consensuada por expertos en el área turística y de alojamiento, para en una fase intermedia ser aplicado a través de una prueba piloto a fin de contar con el instrumento definitivo.

La información fue procesada a través del sistema estadístico de IBM, SPSS. Además, se recurrió a la consulta telefónica para recuperar los datos que faltaban a la vez que se comprobó la información emitida por los establecimientos de forma aleatoria.

La metodología aplicada ha permitido establecer correctamente el estudio de las variables propuestas a los grupos de interés como es el caso de turistas nacionales y extranjeros que arribaron a la ciudad de Cuenca y establecimientos de hospedaje.

\section{RESULTADOS}

\subsection{Oferta Turística}

En cuanto a la existencia de establecimientos de alojamiento el mayor porcentaje corresponde a Hostales con 56\%, luego se encuentran Hoteles con $34 \%$, Hotel Boutique con 4\%, seguido de Hosterías con 3\% y finalmente Casa de Huéspedes y Apartamentos turísticos con $1 \%$. Además, por medio del catastro hotelero del Ministerio de Turismo (MINTUR, 2015) se realizó un cálculo del total de plazas que ofrece el sector de alojamiento cuencano, siendo este de 8.534 plazas diarias (Tabla 1). 
Tabla № 1: Número de plazas ofertadas, de acuerdo al catastro de hospedaje en la ciudad de Cuenca.

\begin{tabular}{|c|c|c|c|c|c|c|c|c|c|}
\hline & \multicolumn{9}{|c|}{ TPO } \\
\hline CATEGORIA & $\begin{array}{c}\text { APARTAMENTOS } \\
\text { TURISTICOS }\end{array}$ & HOSTALES & $\begin{array}{c}\text { HOSTALES } \\
\text { RESIDENCIA }\end{array}$ & HOSTERIAS & $\begin{array}{c}\text { HOTEL } \\
\text { BOUTIQUE }\end{array}$ & HOTELES & PENSIONES & REFUGIOS & Total \\
\hline LUJO & & & & & & 218 & & & 218 \\
\hline PRIMERA & 60 & 1049 & 54 & 337 & 252 & 1226 & 159 & & 3137 \\
\hline SEGUNDA & 70 & 1006 & 310 & 78 & & 1170 & 304 & 12 & 2950 \\
\hline TERCERA & 28 & 798 & 621 & & & 457 & 291 & & 2195 \\
\hline CUARTA & & & & & & 34 & & & 34 \\
\hline Total & 158 & 2853 & 985 & 415 & 252 & 3105 & 754 & 12 & 8534 \\
\hline
\end{tabular}

Fuente: Elaboración propia a partir de los datos del Catastro de hospedaje, MINTUR.

Se generó el cálculo de ocupación de acuerdo a la categoría de los establecimientos de alojamiento, cabe recalcar que el cálculo se realizó desde julio de 2015 hasta diciembre 2016, en el cual se puede observar que los establecimientos de lujo poseen el mayor índice de ocupación (con 42\%) seguidos de los establecimientos de primera con el $35 \%$ de ocupación (Tabla 2).

Tabla № 2: Porcentaje de ocupación hotelera por categoría.

\begin{tabular}{|l|l|l|l|l|l|l|}
\hline Período & Lujo & Primera & Segunda & Tercera & Cuarta & Total \\
\hline Jul-15 & $50 \%$ & $31 \%$ & $29 \%$ & $26 \%$ & $1 \%$ & $29 \%$ \\
\hline Ago-15 & $55 \%$ & $36 \%$ & $26 \%$ & $23 \%$ & $17 \%$ & $29 \%$ \\
\hline Sep-15 & $44 \%$ & $27 \%$ & $23 \%$ & $20 \%$ & $14 \%$ & $24 \%$ \\
\hline Oct-15 & $54 \%$ & $49 \%$ & $28 \%$ & $27 \%$ & & $33 \%$ \\
\hline Nov-15 & $61 \%$ & $56 \%$ & $32 \%$ & $32 \%$ & & $38 \%$ \\
\hline Dic-15 & $55 \%$ & $37 \%$ & $21 \%$ & $24 \%$ & & $27 \%$ \\
\hline Ene-16 & $34 \%$ & $31 \%$ & $22 \%$ & $18 \%$ & & $24 \%$ \\
\hline Feb-16 & $52 \%$ & $40 \%$ & $26 \%$ & $22 \%$ & & $29 \%$ \\
\hline Mar-16 & $48 \%$ & $33 \%$ & $23 \%$ & $13 \%$ & & $24 \%$ \\
\hline Abr-16 & $38 \%$ & $30 \%$ & $16 \%$ & $16 \%$ & $12 \%$ & $21 \%$ \\
\hline May-16 & $35 \%$ & $24 \%$ & $27 \%$ & $27 \%$ & $14 \%$ & $26 \%$ \\
\hline Jun-16 & $19 \%$ & $13 \%$ & $11 \%$ & $12 \%$ & $12 \%$ & $12 \%$ \\
\hline Jul-16 & $30 \%$ & $33 \%$ & $21 \%$ & $20 \%$ & $18 \%$ & $23 \%$ \\
\hline Ago-16 & $23 \%$ & $37 \%$ & $22 \%$ & $25 \%$ & $19 \%$ & $25 \%$ \\
\hline Sep-16 & $25 \%$ & $39 \%$ & $25 \%$ & $25 \%$ & $17 \%$ & $26 \%$ \\
\hline Oct-16 & $65 \%$ & $57 \%$ & $36 \%$ & $37 \%$ & $23 \%$ & $39 \%$ \\
\hline Nov-16 & $21 \%$ & $36 \%$ & $20 \%$ & $24 \%$ & $14 \%$ & $23 \%$ \\
\hline Dic-16 & $35 \%$ & $23 \%$ & $24 \%$ & $18 \%$ & $26 \%$ \\
\hline Total & $30 \%$ & $9 \%$ & $14 \%$ & $50 \%$ & $13 \%$ \\
\hline Fuente- & & & & & \\
\hline
\end{tabular}

Fuente: Elaboración propia a partir de los datos de las encuestas a establecimientos de alojamiento.

Como parte del estudio se determinó el número de personas hospedadas de acuerdo a la categoría a nivel general de los establecimientos hoteleros desde julio de 2015 hasta diciembre de 2016, dando un total de 1.298.008 personas (Tabla 3).

En relación a las necesidades de capacitación que tienen los diferentes establecimientos de alojamiento, se puede apreciar que los hoteles tienen $38 \%$ de capacitación en servicio al cliente, seguido de hoteles boutique con $23 \%$ en cuanto a las necesidades en servicio al cliente, innovación y manejo de página web; finalmente en los hostales una de las mayores debilidades es en servicio al cliente con 41\% (Tabla 4). 
Tabla № 3: Cálculo del número de personas hospedadas.

\begin{tabular}{|c|c|c|c|c|c|c|}
\hline Número de camas & Lujo & Primera & Segunda & Tercera & Cuarta & Total \\
\hline Jul-15 & 3.373 & 30.513 & 26.262 & 17.398 & 11 & $77.557,00$ \\
\hline Ago-15 & 3.705 & 35.416 & 23.321 & 15.660 & 183 & $78.285,00$ \\
\hline Sep-15 & 2.861 & 25.699 & 20.292 & 13.065 & 139 & $62.056,00$ \\
\hline Oct-15 & 3.636 & 47.994 & 25.871 & 18.267 & - & $95.768,00$ \\
\hline Nov-15 & 3.973 & 52.381 & 28.615 & 21.193 & - & $106.162,00$ \\
\hline Dic-15 & 3.691 & 35.964 & 19.259 & 16.597 & - & $75.511,00$ \\
\hline Ene-16 & 2.275 & 30.368 & 20.118 & 11.942 & - & $64.703,00$ \\
\hline Feb-16 & 3.258 & 36.093 & 22.182 & 14.110 & - & $75.643,00$ \\
\hline Mar-16 & 3.215 & 32.417 & 20.809 & 8.693 & - & $65.134,00$ \\
\hline Abr-16 & 2.454 & 28.494 & 14.402 & 10.777 & 124 & $56.251,00$ \\
\hline May-16 & 2.020 & 23.704 & 24.258 & 18.058 & 147 & $68.187,00$ \\
\hline Jun-16 & 2.313 & 16.140 & 8.170 & 9.043 & 510 & $36.176,00$ \\
\hline Jul-16 & 1.271 & 12.236 & 9.865 & 8.453 & 125 & $31.950,00$ \\
\hline Ago-16 & 2.005 & 32.451 & 19.272 & 13.705 & 189 & $67.622,00$ \\
\hline Sep-16 & 1.511 & 34.930 & 19.462 & 16.182 & 198 & $72.283,00$ \\
\hline Oct-16 & 1.716 & 37.900 & 22.444 & 17.047 & 179 & $79.286,00$ \\
\hline Nov-16 & 4.251 & 53.695 & 32.075 & 24.088 & 233 & $114.342,00$ \\
\hline Dic-16 & 1.431 & 34.898 & 18.497 & 16.123 & 143 & $71.092,00$ \\
\hline Total & 48.959 & 601.293 & 375.174 & 270.401 & 2.181 & 1.298 .008 \\
\hline
\end{tabular}

Fuente: Elaboración propia a partir de los datos de las encuestas a establecimientos de alojamiento.

Tabla № 4: Necesidades de capacitación.

\begin{tabular}{|c|c|c|c|c|c|}
\hline & Servicio al cliente & $\begin{array}{c}\text { Innovación de } \\
\text { productos }\end{array}$ & $\begin{array}{c}\text { Manejo de página } \\
\text { web }\end{array}$ & Marketing & $\begin{array}{c}\text { Seguridad e higiene } \\
\text { alimentaria }\end{array}$ \\
\hline Hotel & $38 \%$ & $11 \%$ & $21 \%$ & $19 \%$ & $11 \%$ \\
\hline Hotel Boutique & $23 \%$ & $23 \%$ & $23 \%$ & $15 \%$ & $15 \%$ \\
\hline Hostal & $41 \%$ & $10 \%$ & $21 \%$ & $16 \%$ & $13 \%$ \\
\hline
\end{tabular}

Fuente: Elaboración propia a partir de los datos de las encuestas a establecimientos de alojamiento.

\subsection{Demanda Turística}

Con respecto a la demanda turística se ha creado el perfil del turista que visita Cuenca (Ecuador), con variables importantes como las que a continuación se presentan. La edad del turista que visita Cuenca esta entre los 26 a 35 años el cual está representado por el 29,35\% de encuestados (Gráfico 1).

\section{Gráfico №1: Estructura de edad de los visitantes en \%.}

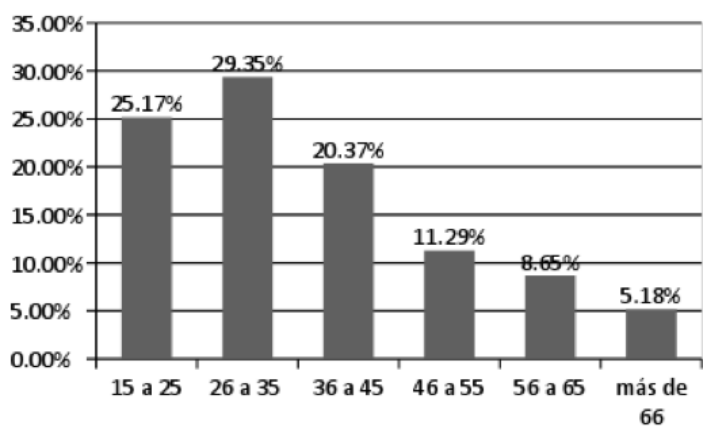

Fuente: Elaboración propia a partir de los datos de las encuestas a turistas nacionales y extranjeros.

Además, se generó la edad de acuerdo a la preferencia de hospedaje, en el cual se resalta que el $40 \%$ del grupo de edad de 46 a 55 años prefieren hospedarse en hoteles, mientras tanto que el 36\% correspondiente a los 26 a 35 se alojan en hostales y finalmente el $4 \%$ que oscila la edad de 36 a 45 años prefieren los hoteles boutique (Gráfico 2).

Gráfico №2: Estructura de edad de los visitantes, por preferencia de hospedaje.

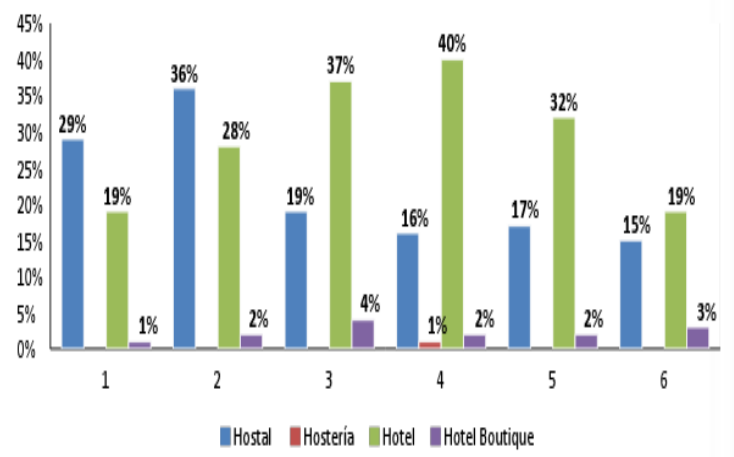

Fuente: Elaboración propia a partir de los datos de las encuestas a turistas nacionales y extranjeros.

En relación al objetivo de la visita se destaca que el 74,30\% de turistas nacionales viajan de vacaciones, recreación y ocio mientras que el 63,84\% de turistas extranjeros tienen el mismo objetivo (Gráfico 3). 
Gráfico №3: Objetivo de la visita a Cuenca (\%del total del conteo por período).

\begin{tabular}{|c|c|c|c|c|c|c|}
\hline \multirow[b]{3}{*}{ Objetivode la visita } & \multirow{2}{*}{\multicolumn{2}{|c|}{2015}} & \multicolumn{2}{|c|}{ Relevamiento / Pais } & \multicolumn{2}{|l|}{0016} \\
\hline & & & & T1 & & 12 \\
\hline & Ecuador & Estados Unidos & Ecuador & Estados Unidos & Ecuador & Estados Unidos \\
\hline Vacaciones, recreación, ocio & $74.30 \%$ & 59.8096 & 48.8996 & $\square_{55.32 \%}$ & $61.21 \%$ & 63.8496 \\
\hline Visita familiares, amigos & 17.3296 & $14.71 \%$ & $22.22 \%$ & $8.51 \%$ & $20.65 \%$ & $11.51 \%$ \\
\hline De compras & $3.35 \%$ & & & & $2.52 \%$ & $1.10 \%$ \\
\hline Radicarse en la ciudad & 2.2396 & $18.63 \%$ & $2.22 \%$ & $14.89 \%$ & $1.26 \%$ & 15.8996 \\
\hline otros & $1.12 \%$ & & |2.22\% & & $0.50 \%$ & $0.27 \%$ \\
\hline Educación y formación & $1.12 \%$ & $1.96 \%$ & $4.44 \%$ & & $4.03 \%$ & 3.849 \\
\hline Negocios y profesiōn & $0.56 \%$ & & $15.56 \%$ & 8.5196 & $5.29 \%$ & $110 \%$ \\
\hline Religión, peregrinación & & 2.9496 & & |6.38\% & 3.0296 & $0.55 \%$ \\
\hline Salud & & $0.98 \%$ & $4.44 \%$ & | $4.26 \%$ & $1.51 \%$ & $0.82 \%$ \\
\hline En tränsito & & $0.98 \%$ & & $2.13 \%$ & & $1.10 \%$ \\
\hline Total general & $100.00 \%$ & 100.0096 & $100.00 \%$ & $100.00 \%$ & 100.0096 & $.000 \%$ \\
\hline
\end{tabular}

Fuente: Elaboración propia a partir de los datos de las encuestas a turistas nacionales y extranjeros.

Los lugares más visitados según los datos recabados en las encuestas muestran que el $62,37 \%$ en el año 2015 prefiere visitar los museos y en el año 2016 el 69,35\% sigue su enfoque de visita a los museos (Gráfico 4).

Gráfico №4: Preferencia por lugares visitados.

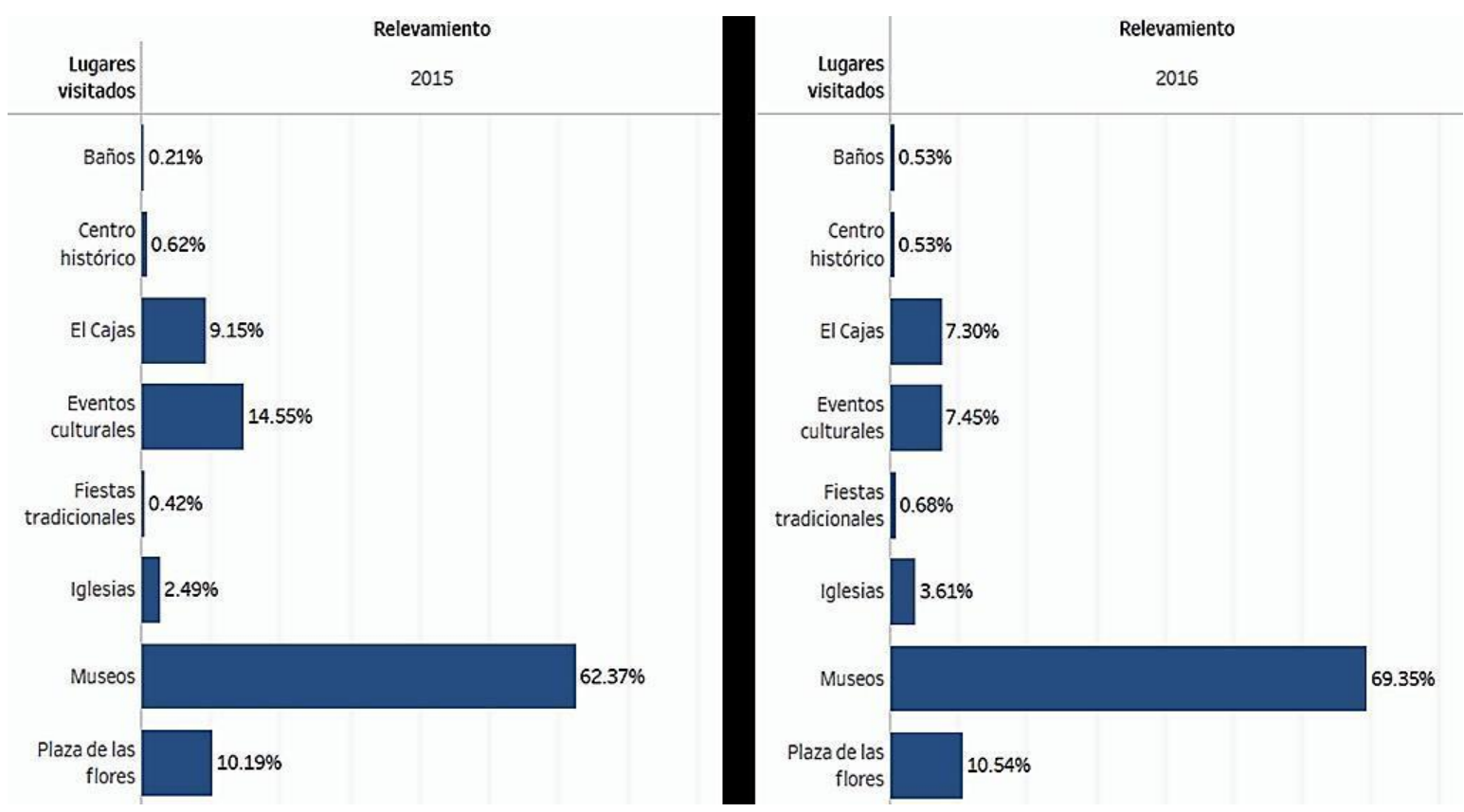

Fuente: Elaboración propia a partir de los datos de las encuestas a turistas nacionales y extranjeros.

El número de noches de estancia promedio de acuerdo a las diferentes categorías de establecimiento de alojamiento y huéspedes nacionales y extranjeros está en un rango entre 2 a 3 noches (Gráfico 5). 
Gráfico №5: Número de noches de estancia promedio de los huéspedes.
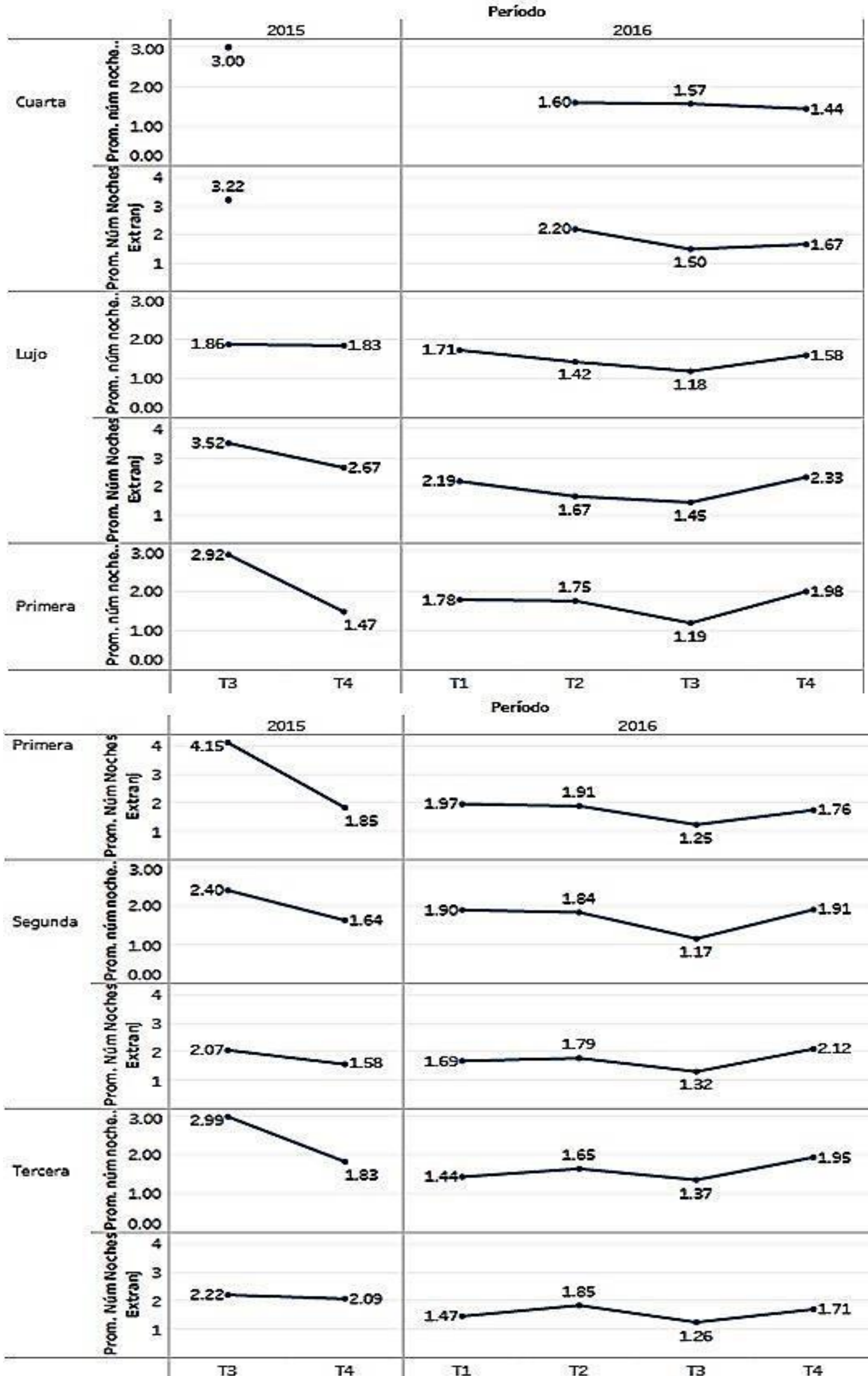

Fuente: Elaboración propia a partir de los datos de las encuestas a turistas nacionales y extranjeros. 


\subsection{Discusión de los Resultados}

Los resultados del estudio de la oferta turística de la ciudad de Cuenca, señalan que en la ciudad la mayoría de establecimientos de hospedaje ofertados corresponde a los hostales con un $56 \%$, seguido por un $34 \%$ de hoteles, y con menor presencia las hosterías y casas de huéspedes o apartamentos turísticos que no superan el 3\%; esto con respecto a las 8.534 plazas diarias que se ofrecen en la ciudad (MINTUR, 2015) con que se dispone para responder al crecimiento constante en el volumen de arribos internacionales al país, el cual supera el millón y medio de visitantes (Santiago, Romero, \& Álvarez, 2017) y que constituyen el potencial mercado para la ciudad.

Por su parte, el porcentaje de ocupación de esta oferta hotelera, por categoría, corresponde a los establecimientos de lujo con $42 \%$ seguidos de los establecimientos de primera categoría con el 35\% de ocupación; con lo cual se puede indicar que la oferta de alojamiento en Cuenca guarda relación con la tasa de ocupación promedio para instalaciones hoteleras a nivel nacional que corresponde al alojamiento de primera categoría en un 43\%, mientras para las instalaciones hoteleras de lujo ascendió al 65\% para el año 2016, con un ingreso promedio que oscila entre 60 y 90 por habitación (Santiago, Romero, \& Álvarez, 2017). Estos establecimientos de lujo y de primera categoría corresponden generalmente a cadenas hoteleras que desde la segunda mitad del siglo XX ha venido reemplazando paulatinamente a los hospedajes pequeños e independientes (Jímenez, 2008).

El número de personas hospedadas a nivel general de los establecimientos hoteleros desde julio de 2015 hasta diciembre de 2016 ha alcanzado un total de 1.298 .008 personas, siendo el año 2016 menor al 2015 (considerando los mismos meses para cada año). Esto concuerda con la situación similar que se vivió a nivel nacional donde los ingresos que se alcanzaron el año 2016 ascienden a 1075.5 millones de USD, inferior a los 1173.8 millones del año 2015 (Santiago, Romero, \& Álvarez, 2017), evidenciando que hubo una importante baja en el año 2016 tanto en número de personas hospedadas como en ingresos económicos generados, lo cual, se podría deberse al terremoto ocurrido en abril del 2016 que afectó al turismo del país.

En relación a las necesidades de capacitación que tienen los diferentes establecimientos de alojamiento en primer lugar se ubican necesidades de capacitación en servicio al cliente (34\% promedio), y, en segundo lugar, capacitación en manejo de página web (22\% promedio), si se observa los resultados generales de la tabla. Esto debido al evidente e incesante desarrollo de las tecnologías de la información y la comunicación y su aplicación al turismo que han provocado un cambio en las funciones tradicionales de producir, comercializar y proporcionar el servicio; así como la aparición de una demanda cada vez más exigente, experimentada y compleja que exige mejores servicios (Flores \& Barroso, 2012) tanto en la atención como en la aplicación tecnológica. De ahí la necesidad de los establecimientos hoteleros de contar con una página web y con personal capacitado para manejarla optimizando este recurso y aprovechando sus ventajas para enfrentar el mercado turístico actual.

En cuanto a la variable demanda turística, los resultados muestran que el número de noches de estancia promedio de acuerdo a las diferentes categorías de establecimiento de alojamiento y huéspedes nacionales y extranjeros está en un rango entre 2 a 3 noches que es un tiempo corto de viaje que permite definir que Cuenca es un destino de paso que generalmente está acompañado, y complementa, la visita a otras ciudades del Ecuador, lo cual se facilita puesto que es un país pequeño que se ha caracterizado por ser un destino en que el turista puede visitar varios lugares y diferentes zonas climáticas en poco tiempo. Así, las características del destino han disminuido la estancia del turista en el destino, aumentando su número de salidas, evidenciando una tendencia de movimiento mundial de millones de personas en cortos períodos de tiempo que ha incidido en la aparición de turoperadores que organizan viajes completos en formato de paquetes turísticos (Flores \& Barroso, 2012).

Con respecto a la edad del turista que visita Cuenca esta entre los 26 a 35 años, que representa a turistas jóvenes, profesionales que los convierte en una demanda más experimentada, exigente y ávidos que experiencias turísticas novedosas, que dan lugar a la aparición de nuevos productos turísticos y nuevas tipologías de turismo (Flores \& Barroso, 2012).

En relación al objetivo de la visita se destaca que el $74,30 \%$ de turistas nacionales viajan de vacaciones, recreación y ocio mientras que el $63,84 \%$ de turistas extranjeros tienen el mismo objetivo; lo cual, guarda relación con una tendencia global hacia el turismo recreacional, que ha surgido como consecuencia, entre otros, por el reconocimiento de los gobiernos 
del derecho a unas vacaciones remuneradas para los trabajadores (Flores \& Barroso, 2012) que favorece al turismo debido al incremento de movilidad de las personas con intereses de conocer nuevos destinos para descansar.

Finalmente, los lugares más visitados con el $62,37 \%$ en el año 2015 refiere a las visitas de museos, una tendencia que se mantiene el año 2016 con el $69,35 \%$ de visitas a museos. Tal como se ha mencionado, el turismo es un fenómeno social y cultural que implica la movilidad humana frente a la necesidad de libertad para conocer el mundo e interactuar cara a cara con otras culturas (Dachary, 2015; Campodónico, 2016), y la cultura del mundo genera una espiral de diversificación de las experiencias de consumo (Dachary, 2015); de ahí que se prefieran visitar ciudades culturales, especialmente si han sido calificadas internacionalmente como patrimonio cultural, cuya memoria habita en los museos.

\section{CONCLUSIONES}

La implementación de indicadores turísticos en una ciudad Patrimonial como lo es Cuenca, es de importancia por el hecho que se necesita hacer proyecciones de la situación del sector turístico, cabe mencionar que la ciudad cuenta con estadísticas turísticas débiles.

Mediante el estudio realizado se ha definido que la edad del visitante esta entre los 26 a 35 años, la preferencia de hospedaje está entre hoteles y hostales, el objetivo de visita principalmente se centra en vacaciones, recreación y ocio, la preferencia de lugares visitados son los museos y finalmente el promedio de estancia de acuerdo a los huéspedes nacionales y extranjeros está en un rango de 2 a 3 noches.

Finalmente se alcanzaron resultados favorables obteniendo una alianza estratégica con el sector privado como son: Asociación Hotelera del Azuay, Asociación de Bares y Restaurantes del Azuay y la Cámara de Turismo del Azuay con quienes se firmó un acta de compromiso para acceder a los diferentes establecimientos hoteleros y de restauración del cantón Cuenca para continuar con el estudio y aplicación de indicadores, además se inició la creación de un boletín estadístico anual como herramienta de conocimiento divulgativo y finalmente generó la vinculación de la academia en los procesos de investigación como factor clave en los procesos de tomas de información.

\section{REFERENCIAS}

Ayaviri, V., Quispe, G., \& Sánchez, P. (mar de 2017). Estimación de la demanda del turismo en la Comunidad Andina de Naciones. Revista Espacios, 38(31), 24-38. Recuperado el 17 de ene de 2018, de http://www.revistaespacios.com/a17v38n31/a17v38 n31p24.pdf

Boullón, R. C. (2006). Planificación del espacio turístico.

Campodónico, R. (jun de 2016). Turismo: de la movilidad al espacio. Revista Latino-Americana de Turismología, 1(2), 8-16. Recuperado el 18 de ene de 2018, de $<$ https://rlaturismologia.ufjf.emnuvens.com.br/rlaturis mologia/article/view/50/20>.

Dachary, A. (dic de 2015). El turismo: un modelo de desarrollo. Revista Latino-Americana de Turismología, 1(1), 16-26. Recuperado el 17 de ene de 2018, de https://rlaturismologia.ufff.emnuvens.com.br/rlaturis mologia/article/view/17/2

Departamento Administrativo Nacional de Estadística. (2017). Encuesta de Viajreso Internacionales. Obtenido de http://www.dane.gov.co/files/investigaciones/boletin es/EVI/bol_EVI_sep_12_ago13.pdf

Flores, D., \& Barroso, M. de la O. (2012). La demanda turística internacional. Medio Siglo de evolución. Revista de Economía Mundial, (32), 127-149. Recuperado el 16 de ene de 2018, de http://www.redalyc.org/pdf/866/86625395006.pdf

Fundación Municipal Turismo para Cuenca \& Ministerio de Turismo. (2011). Plan Estratégico de Desarrollo Turístico del Cantón Cuenca y su Área de Influencia. Obtenido de https://cuenca.com.ec/sites/default/files/01.\%20Plan\% 20Estrategico\%20de\%20Desarrollo\%20Tur\%C3\%ADsti co.pdf

Fundación Municipal Turismo para Cuenca. (2017). Patrimonio Cultural y Reconocimientos. Obtenido de http://cuenca.com.ec/es/conoce-cuenca

Instituto Costarricense de Turismo. (2017). Anuario Estadístico de Turismo. Obtenido de http://www.ict.go.cr/es/estadisticas/informesestadisticos.html

Ivanov, S., \& Webster, C. (2007). Measuring the impacts of tourism on economic growth. Tourism Economics, 13(3), 379-388. doi:https://doi.org/10.5367/000000007781497773

Jímenez, A. (2008). Las cadenas hoteleras en el mundo y evolución de su operación en México al inicio del siglo XXI. Innovar, 18 (32).

Ministerio de Comercio Exterior y Turismo. (2017). Reportes Estadísticos de Turismo. Obtenido de http://www.mincetur.gob.pe/turismo/reportesestadisticos-de-turismo/

Ministerio de Turismo. (2013). Cuenca y Quito candidatas para las "7 ciudades maravillas del mundo". Obtenido de http://www.turismo.gob.ec/cuenca-y-quito-candidataspara-las-7-ciudades-maravillas-del-mundo/ 
Ministerio de Turismo. (2017). Anuario de Estadísticas Turísticas. Retrieved from Ministerio de Turismo

MINTUR. (2015). Catastro Hotelero. Cuenca, Azuay, Ecuador. Organización Mundial del Turismo. (2004). National and Regional Tourism Planning: Methodologies and Case. Madrid: OMT.

Organización Mundial del Turismo. (2013). Entender el turismo: Glosario Básico. En O. M. Turismo, Notas metodológicas de la base de datos de estadísticas de turismo (pág. 19). Madrid: UNWTO.

Saeteros, A., Da Silva, E., \& Calles, V. (jul de 2017). Análisis de la Demanda del Turismo para la Gestión Sustentable del Destino en las Islas Galápagos-Ecuador. Anais Brasileiros de Estudos Turísticos - ABET, 7(1), 78-88. Recuperado el 18 de ene de 2018, de https://abet.ufff.emnuvens.com.br/abet/article/view/ 2973.

Santiago, N., Romero, A., \& Álvarez, G. (jul de 2017). Actualidad y proyecciones de desarrollo del turismo internacional en Ecuador. Revista de Ciencia, Tecnología e Innovación, 4(3), 276-287. Recuperado el 16 de ene de 2018, de 186.46.158.26/ojs/index.php/EPISTEME/article/view/7 21

Wallingre, N. (2005). La necesidad de implementar una cultura organizacional innovadora en las Pymes hoteleras de Argentina. Tiempo de Gestión, 84-93.

Zhao, W., \& Li, X. (2006). Globalization of Tourism and Third World Tourism Development - A Political Economy Perspective. Chinese Geographic Science, 16(3), 203210. https://doi.org/10.1007//s11769-006-0203-2.

\section{Processo Editorial / Editorial Process}

Editor Chefe/Editor-in-chief: PhD Thiago D. Pimentel (UFJF).

Recebido em 20 de Novembro de 2017; aceito em 10 de Janeiro de 2018; publicado online 05 de Fevereiro de 2018. Received on November 20, 2017; accepted on January 10, 2018, published online on February 05, 2018. Texto original/ Original paper. Sistema de revisão cega por pares / Double blind review system. 